\title{
All-Optical VCSEL-to-VCSEL Injection Based on Cross Gain Modulation for Routing in Multinode Flexible Spectrum Optimization in Optical Fibre Transmission Links
}

\author{
P. P. Dlamini $\mathbb{D}^{1},{ }^{1}$ G. M. Isoe, ${ }^{1}$ D. Kiboi Boiyo, ${ }^{2}$ A. W. R. Leitch, ${ }^{1}$ and T. B. Gibbon ${ }^{1}$ \\ ${ }^{1}$ Centre for Broadband Communication, Physics Department, Nelson Mandela University, P.O. Box 77000, \\ South Campus, Port Elizabeth-6031, South Africa \\ ${ }^{2}$ Physics Department, Machakos University, P.O. Box 136, Machakos 90100, Kenya
}

Correspondence should be addressed to P. P. Dlamini; s210140313@mandela.ac.za

Received 3 April 2019; Revised 27 May 2019; Accepted 14 July 2019; Published 4 August 2019

Guest Editor: Surinder Singh

Copyright (c) 2019 P. P. Dlamini et al. This is an open access article distributed under the Creative Commons Attribution License, which permits unrestricted use, distribution, and reproduction in any medium, provided the original work is properly cited.

\begin{abstract}
In this paper, we experimentally present a novel, all-optical spectral efficient vertical-cavity surface-emitting laser- (VCSEL-) based technique for routing and spectrum assignment in optical networks. Exploiting all optical VCSEL-to-VCSEL injection to attain cross gain modulation, the optical transmitter is optimized for optical transmission paths to assure quality of service by overcoming blockage for differentiated bandwidth demands during network congestion incidences. A 10 Gbps directly modulated $1549 \mathrm{~nm}$ master VCSEL is optically injected into the $1549 \mathrm{~nm}$ side modes of a $1550 \mathrm{~nm}$ slave VCSEL. The Shannon limit is considered for higher transmission rates with the problem decomposed into degraded routing and spectrum assignment and chromatic dispersion in the optical transmission link penalties. In this work, the proposed technique achieved a $1.3 \mathrm{~dB}$ penalty for transmission over a $25 \mathrm{~km}$ G.655 nonzero dispersion-shifted single-mode optical fibre, a value within the transmission media and optical system characteristics of $3 \mathrm{~dB}$ as recommended by the International Telecommunication Union-Telecommunication (ITU-T). The number of transceivers, switches, and optical transmission links in the network was reduced, increasing the number of satisfied bandwidth requests, thus optimizing the spectral resource utilization.
\end{abstract}

\section{Introduction}

The exponential growth in the global IP traffic volume forces the optical transport network to develop new technologies, upgrading the conventional grid dense wavelength division multiplexing (DWDM) systems to not only sustain the demand for bandwidth but also offer real-time resource management for the network. The traffic explosion is associated with the emerging cloud-based hosted applications such as 5G social networking, remote learning, and Internet of things. These require higher capacity for the bandwidth demands and dynamic services at a reduced cost per bit. This has led to extensive research on optimizing the network resources in the optical transport network and the quality of service. Thus, network operators are continuously finding ways to upgrade the systems to match the ever-increasing demands for global communication, enlarging the capacity without neglecting the effects they have on the quality of service in a cost-efficient manner. In the past, the DWDM optical network promised to be a solution for the traffic growth: dividing the available transmission bandwidth of an optical fibre into nonoverlapping wavelengths with a fixed grid spectrum channel spacing typically ITU-T 50 or $100 \mathrm{GHz}$ [1]. These networks offer the possibility to establish wavelength connections at a fixed bit rate, i.e., 10, 40, and recently $100 \mathrm{~Gb} / \mathrm{s}$ at $50 \mathrm{GHz}$ channel spacing [2]. The bandwidth request of today's network end user requires more customized services and more differentiated demands with different prices [3]. This makes it challenging to upgrade the DWDM-based systems to adapt to changing traffic and network conditions, as the flexibility is limited to the transmitter frequency tunability and the reconfiguration 
allowed by the optical switching nodes. The term "flexibility" refers to the ability of the network to dynamically adjust the optical fibre bandwidth and modulation format according to the request of each connection for efficient resource utilization [4]. This is of vital importance as the utilized bandwidth of the optical fibre is reaching its maximum limit with the capacity crisis approach with current networks attempting to accommodate the ever-increasing and dynamic traffic volume. In recent years, the flexible spectrum network has received great attention as it efficiently utilizes the available spectrum in an optical fibre compared to the fixed grid systems. One of the advantages of the flexible spectrum network is that the optical spectrum is divided into finer granular frequency slots of $12.5 \mathrm{GHz}$ whose full width can be dynamically adjusted to create arbitrary densely packed sub- and superchannels offering a tunable bit rate from 10 to $100 \mathrm{~Gb} / \mathrm{s}$ and beyond [5-7]. This is a technique used in the industry to overcome the theoretical maximum transmission rate of a channel for a particular noise level (Shannon limit) [8]. This push in the transmission rates of a single optical carrier brings the limit for transmission over any meaningful reach. Other advantages of flexible spectrum networks include reconfigurable nodes, bandwidth-variable transmitters, adjustable wavelength and spectrum allocation, and the centralized network management $[9,10]$.

This enables routing according to the traffic volume and spectrum assignment according to the bandwidth request. Superchannels in the flexible spectrum transmission link address how the rates can be increased without any consideration of the other challenge faced by the optical network, hence maximizing fibre capacity. Therefore, not only should the finer spectral granularity (dependent on the channel baud rate) be considered but also the finer spatial granularity which relates to the grouping of single mode fibres. These must be adaptable with respect to the traffic volume and be reconfigurable to the bandwidth request (spectrum assignment and routing) for globally optimum resource utilization with focus on the transmission, medium, and receiver [11]. The industry is moving to flexible spectrum allocation schemes that support arbitrary channel width as opposed to $50 \mathrm{GHz}$; this is because of the advances in tunable technologies such as VCSELs [12]. Multinode networks can be considered by joining multiple point-to-point links of superchannel signals using flexible spectrum routing and channel assignment. This should combine colourless, directionless, and contensionless reconfigurability to route individual wavelengths across fibres over multiple nodes without optical-electronic-optical conversion achieving power and cost efficiency [13]. Maintaining an end-to-end source to destination communication and quality of service which is mainly affected by linear and nonlinear fibre impairments is a major challenge for reconfigurable flexible spectrum networks. These optical layer impairments include cross talk, four-wave mixing, scattering, polarization mode dispersion, chromatic dispersion, and attenuation [14]. Network designers implement components that are cheaper in cost with reduced operational expenses and low power consumption.

A conventional fixed ITU grid DWDM optical network can be upgraded to have the features of a reconfigurable elastic spectrum by using a wavelength converter configuring a multinode spectrum network. In wavelength conversion, a signal at a given wavelength is transferred to another wavelength in order to be routed on a different optical connection and release the original wavelength resource to another signal [15]. For a fixed set of wavelengths, the conversion can be used to switch from one channel to an adjacent channel as a spectral efficient technique. There are a number of converters that have been reported which include optoelectronic, laser, coherent, and controlled optical gates. In this work, we for the first time to our knowledge propose a routing and spectrum assignment technique through reallocation of central frequency of the optical transmitter, as a means to increase the probability of finding a sufficient continuous spectrum $[16,17]$. This is to improve network resource utilization and capacity and resolve wavelength contention associated with a flexible spectrum in optical communication networks. The technique used is an alloptical wavelength conversion cross gain modulation (XGM) approach for a directly modulated wavelengthtunable vertical-cavity surface-emitting laser (VCSEL). A major attribute of all-optical wavelength converters is the ability to tune the wavelength of the laser during the conversion. This makes tunable lasers an essential component for future flexible spectrums with application to wavelength switching, routing, wavelength conversion, and reconfigurable optical add-and-drop multiplexer (ROADM) for multinode optical networks $[15,18]$. VCSELs lasing at $1550 \mathrm{~nm}$ with $10 \mathrm{Gbps}$ are commercially available. These offer low manufacturing cost as construction enables the use of traditional semiconductor-manufacturing techniques. The manufacturing yield is increased because of the capability for efficient testing at the wafer level [19]. VCSELs easily and inexpensively provide wavelength tuning over a relatively large range by varying the bias current. They further emit a highly circular beam that can be coupled into the optical fibre with very high efficiency [20].

1.1. Optical Fibre Impairments and Transmission Standards in Multinode Networks. All optical networks with routing and wavelength assignment capability have received extensive attention in flexible spectrum systems as they support dynamically varying traffic volume, managing the spectral resources efficiently. This can be achieved by having centralized network management in a unified softwaredefined networking (SDN) controller platform responsible for channelization, bandwidth allocation, routing and wavelength assignment, end-to-end fibre connectivity, and service restoration in case of fibre faults in any link [21]. When planning the configuration of the flexible spectrum network, the designer must take topology, traffic matrix, and physical layer models into consideration as inputs. A typical multinode network consists of a transmitter/source (node 1) connected to the receiver/destination (node 3 ) via DWDM fibre links that propagate channels through colourless, directionless, and contensionless reconfigurability to route individual wavelengths from fibre to fibre across multiple nodes, as shown in Figure 1. 


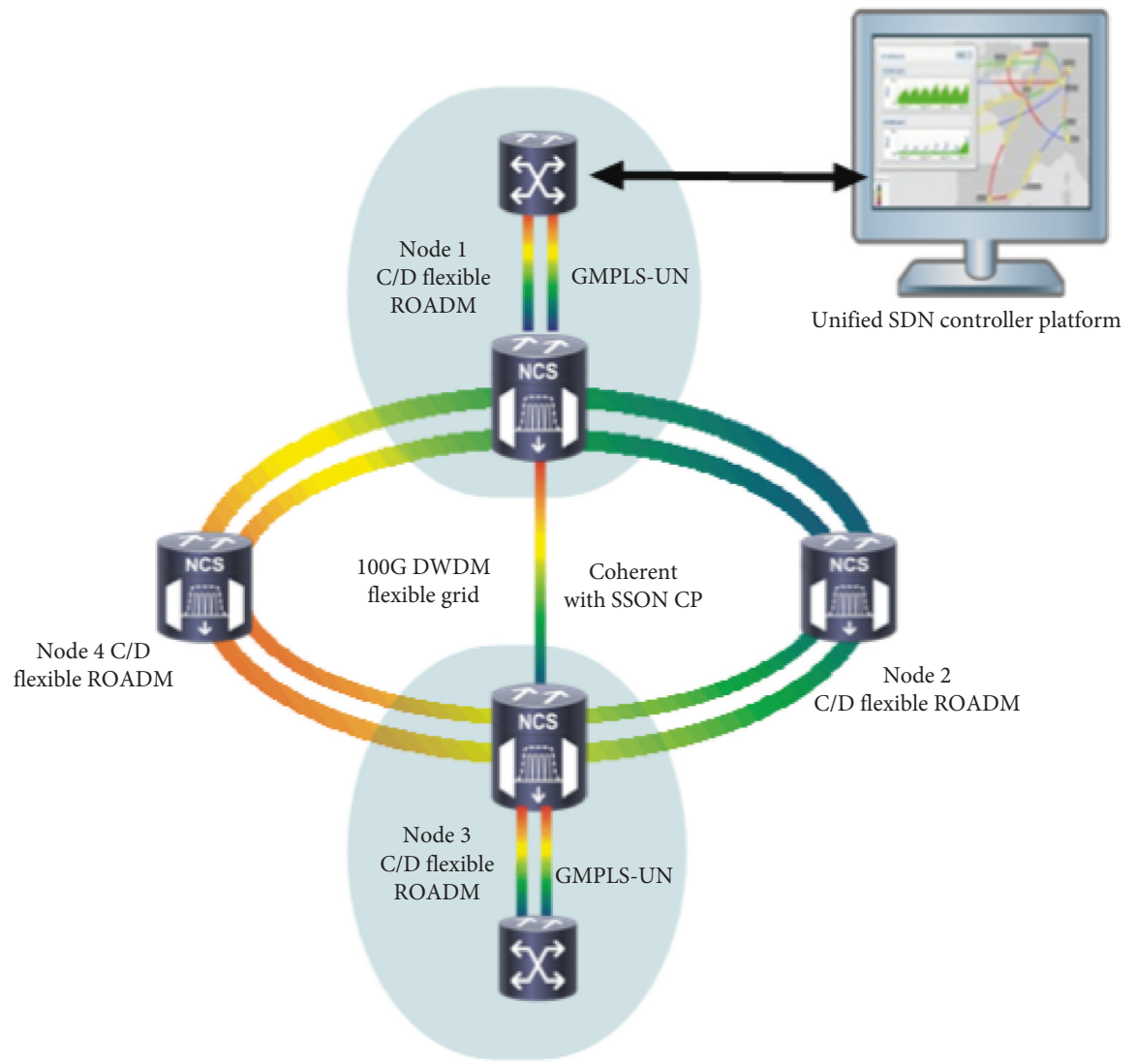

Figure 1: Simple topology of the multinode network used for the experimental demonstration of bandwidth utilization for the flexible bandwidth network [21].

These nodes have wavelength selective switch capability and a ROADM which can be used to convert optical signals from one wavelength to another for spectral efficiency and routing the user demands through spatial efficient lightpaths. This means that multiple channels are transmitted using variable bandwidth transceivers through reach and capacity optimized links for each of the many arbitrary channels generated using one of the many possible modulation formats [20-23]. Thus, the spectral and spatial flexibility in optical transport networks can be optimized in the network management system (SDN), controlling the modulation level of a channel according to maximized spectral efficiency while maintaining the required quality of service [24]. Optical signals with high spectral efficiency are highly sensitive to physical layer impairments; this is seen as a bit error rate rise or degradation of the signal-to-noise ratio of the affected optical link.

Our aim is to put together an effective optical performance-monitoring method for a routing and wavelengthassigning flexible bandwidth multimode network that can offer adaptive optimization of the lightpaths based on the known impairments. When discussed that all lightpaths of a flexible spectrum are assumed to have adequate quality of service for a given signal which gets affected by the linear and nonlinear impairments, the optical transport system needs to be optimized in terms of receiver sensitivity and extinction ratio as a function of bias current and drive current for a known modulation format, bit rate, and laser launch power. This is useful in understanding the quality of service achievable by different modulation conditions of transmitters and the corresponding sensitivity expected at the receiver and can be further analyzed considering the impact of the fibre characteristics through all-optical conversion through cross gain modulation power injection. To properly size the spectrum for each end user demand based on its bit rate and transmission distance, a bandwidth-variable transmitter can be used to adjust the modulation format such that it occupies less optical spectrum whilst still performing error-free transmission due to reduced impairments. This is to maximize the reach (fibre length), effectively utilize the network resources, and broaden the spectral width (routing, wavelength assignment, and channel switching). With the demand for bandwidth of internet-based emerging applications, high-speed networks have been receiving extensive research interest. As the bit rate increases, it is necessary to increase the launch power, making the impairments a stringent hindrance. Nonlinear impairment effects generate on each channel and also crosstalk between channels as discussed in [14]. The linear impairment effects are independent of signal power affecting wavelengths individually, and these will be focused on in this work as they are not uniform and depend on the node spacing and the characteristics of the installed fibre. Single mode fibres are used for high-bandwidth and long-distance 
transmission, and these have a much smaller core size and no inherent distance limitations caused by modal dispersion [25]. These come in many different formulations such as low water peak fibre (LWPF), dispersion-shifted fibre (DSF), nonzero dispersion-shifted fibre (NZDSF), and bend insensitive fibre.

In this work, SRS G.655 nonzero dispersion-shifted fibre (NZDSF) was used for transmission as it provides optimized performance for the multichannel DWDM optical transport network with efficient resource utilization at lowest total system cost $[25,26]$. It offers low dispersion slope, dispersion value, and effective area to accommodate the current lower channel count and the future full band capabilities for networks operating in the traditional C-band (1530$1565 \mathrm{~nm})$ and the emerging L-band (1565-1625 nm), providing lowest residual dispersion [27]. This is an important requirement for optical fibres forming the transport system on the bandwidth demanding requests of end users of nextgeneration applications such as multinode all-optical flexible networks based on cross gain modulation capable of routing and wavelength assignment at $10 \mathrm{~Gb} / \mathrm{s}$. Other benefits of SRS G.655 NZDSF include easiness, less expensive dispersion compensation (uses of reverse dispersion fibres), low polarization mode dispersion, and low attenuation at the water peak.

These values describe specifications for polarization mode dispersion (PMD) and the transmission attributes of the single mode fibre which has an absolute value of chromatic dispersion (CD) coefficient greater than some nonzero values throughout the $\mathrm{C}$-band wavelength range. This is to satisfy the objectives of a network based on the PMD requirements and CD characteristics to provide information about the transmission distance and bit rate. PMD values depend on the geometrical and mechanical condition of the fibre; for ITU-T G.655, a maximum PMD link design value of $0.2 \mathrm{ps} / \sqrt{ } \mathrm{km}$ is specified [28]. The chromatic dispersion in $\mathrm{ps} / \mathrm{nm}$ can be calculated from the chromatic dispersion coefficients of the factory lengths, assuming a linear dependence on length, and with due regard for the signs of the coefficients, for SRS G.655 NZDSF, a maximum chromatic dispersion of $-2.8 \mathrm{ps} / \sqrt{ } \mathrm{km}$ at $1550 \mathrm{~nm}$ is specified. The fibre had a typical loss of $0.2 \mathrm{~dB} / \mathrm{km}$ and a dispersion of $17 \mathrm{ps} / \mathrm{nm} / \mathrm{km}$ at $1550 \mathrm{~nm}$. Based on these impairments affecting the flexible spectrum network, an alloptical performance network method may be achieved for the optimization for known standards [28, 29]. This can be used as a real-time performance method to maximize spectral efficiency while maintaining the required quality of service, efficient resource utilization, and bit error rate performance. This will be compared with the transmission penalty for a $50 \mathrm{~km}$ metro system which is $4 \mathrm{~dB}$ measured at the $10^{-9}$ BER threshold. For a VCSEL of launch power $11 \mathrm{dBm}$, an ER of $13.7 \mathrm{~dB}$ is the upper limit and $8 \mathrm{~dB}$ is the lower limit for backto-back transmission [30].

\section{Proposed Routing in Multinode Flexible Spectrum Technique}

Wavelength reassignment and routing are some of the features of the flexible spectrum which are of importance in multinode all-optical networks. Optoelectrical converters are capable of achieving function by retransmitting incoming signals that have been detected in a new wavelength. The major drawback of such devices is the large power consumption and electronic circuit complexity in highspeed networks operating at $10 \mathrm{Gbps}$ and beyond. There are coherent converters that can handle all signal modulation formats such as four-wave mixing using semiconductor optical amplifiers (SOAs) which are dependent on the output wavelength of both the pump and the input signal [22]. This limits flexibility degree of freedom which is essential in multimode networks, and a tunable pump will be required to even achieve wavelength conversion/reassignment at fixed output channels. A simpler all-optical wavelength conversion technique has been demonstrated. The conversion is performed by controlling a single master laser, launching its input signal into a tunable wavelength laser, resulting in gain saturation used to manage the laser oscillation. The laser resonance frequency limits the speed, and this is a major drawback for future optical networks expected to operate at speed beyond $10 \mathrm{Gbps}$ [23]. In cross-phase and cross-gain modulation modes, semiconductor amplifiers are used as optically controlled gate converters. In cross-phase modulation, the advantage of the converters is the dependency of the carrier density on the active region of the amplifier [24]. The technique has drawbacks in systems needing intensity-modulated output signals which will require an interferometer as the output signal is phase modulated. In cross gain modulation converters, the data can be transferred from one wavelength to another without optical-electronic-optical conversion $[25,26]$. This makes the cross gain modulation technique a simple and cost-efficient all-optical conversion. In this work, this was used for flexible spectrum optimization and wavelength switching as discussed below.

\subsection{All-Optical Wavelength Conversion Cross Gain Modulation} (XGM). For all-optical wavelength conversion from one wavelength to a different adjacent wavelength for intensitymodulated signals, cross gain modulation is the most practical technique as it is insensitive to the polarization of the signal. The technique is based on gain saturation, as shown in Figure 2, in which the input signal modulates the gain in the semiconductor optical amplifier.

Gain variation of an input signal carrying information is used to modulate a signal at the desired output wavelength, such that these data are transferred with the exception of them being inverted. The conversion speed is determined by carrier dynamics governed by the relative slow interband carrier recombination [31]. The conditions needed to operate the semiconductor optical amplifier are large current injection and high optical power in order to achieve high bit rate conversion $[22,32]$. Since the injection current is limited, the conversion speed can thus be increased by using longer laser cavity lengths. In multinode flexible spectrum networks, each node has the capability to convert an incoming wavelength to a new wavelength for reassignment and rerouting based on the demands of the end user. Cross 


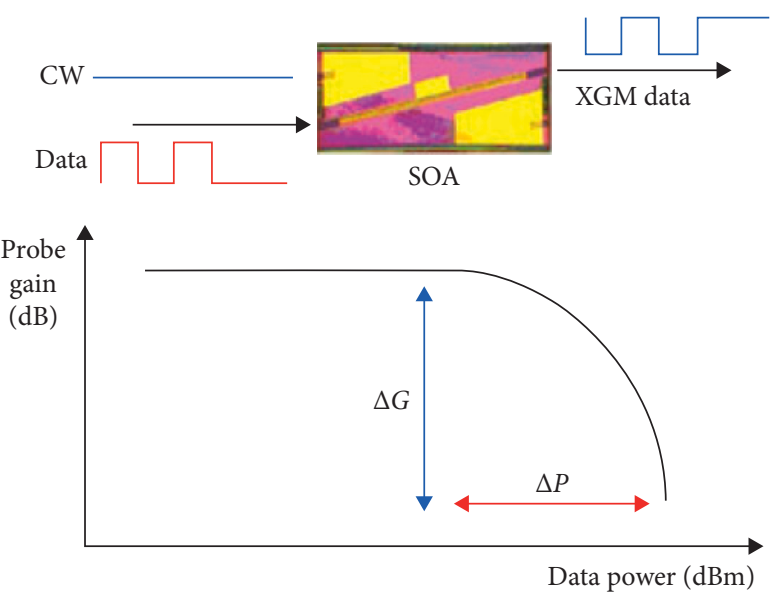

FIGURE 2: Optical wavelength conversion cross gain modulation (XGM) operation principle [31].

gain modulation makes it possible to switch the data signal from one wavelength to another through all-optical power injection which minimizes the number of components in the network, hence lowering the overall operation costs of the system. To keep up with expectations of the future optical network such as the wavelength reassignment and lightpath rerouting, the technique can be integrated with components of high-speed networks so as to increase the capacity and reach of metro system transmissions.

With the conditions of operation of the semiconductor optical amplifier for high bit rate conversion already stated, the lasers to be used for optical power injection were first characterized. The trade-off between the bias current and the output power was experimentally demonstrated for two tunable VCSELs, as shown in Figure 3.

The wavelength of a VCSEL can be tuned by varying the bias current, as shown in Figure 3(b). This characteristic is seen for both VCSELs, emitting different wavelengths at different bias currents. The VSCEL cavity is current driven; each bias point corresponds to a different wavelength for current after the lasing point and before saturation which is typically $1 \mathrm{~mA}$ and $7 \mathrm{~mA}$, respectively. The wavelength can be tuned at fixed channel spacing with each VCSEL covering a finite band. The two bands formed an overlap such that the wavelength coverage is continuous [33]. The band coverage of one VCSEL was characterized at different bias points tuned at fixed channel spacing with a different wavelength output represented by different colours, as shown in Figure 4(a).

The second VCSEL was selected such that the wavelength bands of the two VCSELs overlap so as to maximize the total spectral bandwidth. This improves network resource utilization and resolves wavelength contention associated with the flexible spectrum. The total continuous spectral bandwidth of the system was increased, as shown in Figure 4(b). The transmission performance of a VCSEL depends not only on its bias current, but the drive current/modulation index is also a factor and should be considered [33]. Despite wavelength tunability and increased spectral bandwidth of the VCSEL, certain operating points may give rise to unacceptable penalties. Hence, the quality of the signal was investigated at given bias points for different drive currents. This was done to quantify the extinction ratio and receiver sensitivity of the network for optimized transmission performance. All-optical wavelength conversion through cross gain modulation was used to seamlessly tune across the combined spectral bandwidth of the two VCSELs. The bands formed have the side modes of slave laser wavelength overlapping with the dominant modes of the master laser. The intensity-modulated master VCSEL is optically power injected to the side mode of the slave VCSEL. This results in temporally switching "OFF" (suppressing) the dominant mode, while the side mode is switched "ON" (gain), and vice versa [31]. As a result, wavelength conversion takes place with data transferred from one wavelength (already received) to another wavelength (that was not being used). The slave VCSEL transmits the inverted data stream of the master VCSEL. This allows the converted data to be routed and the new wavelength be assigned to the next node for transmission in multinode flexible spectrum networks.

\section{Experimental Setup}

Wavelength collision negatively affects resource utilization in flexible spectrum optical networks as it reduces the number of usable wavelength bands in the available spectral bandwidth. Various wavelength conversion techniques have been presented in WDM systems to effectively minimize this effect without assigning extra bandwidth by adding more components. The Centre for Broadband Communication research group at Nelson Mandela University has experimentally demonstrated an all-optical VCSEL-to-VCSEL wavelength conversion technique for transmission at a low attenuation $1550 \mathrm{~nm}$ window [34]. The technique was further studied and used to experimentally demonstrate all-optical injection for spectral efficient utilization through defragmentation and wavelength switching on a cascade of vertical-cavity surface-emitting lasers successfully. This was explored further for transmission application in nodes capable of routing and wavelength assignment in multinode flexible networks, as shown in Figure 5.

A 10 Gbps $1550 \mathrm{~nm}$ single mode VCSEL 1 (node 1) with a wavelength tunability range of $5.2 \mathrm{~nm}$ was directly modulated at $8.5 \mathrm{Gbps}$ by a non-return-to-zero (NRZ) pseudorandom binary sequence (PRBS $2^{7}-1$ ) from a programmable pattern generator (PPG). The PPG was used to vary the modulation conditions to match the desired drive current. Biased at $5 \mathrm{~mA}$ with a drive current of $5 \mathrm{~mA}$ lasing at $1548.20 \mathrm{~nm}$ wavelength with $-3 \mathrm{dBm}$ optical output power, the master laser was optically injected to the side mode with a similar wavelength of VCSEL 2 biased at $9.5 \mathrm{~mA}$ current with a dominant mode lasing at $1550.35 \mathrm{~nm}$ with an optical output power of $-4.49 \mathrm{dBm}$. To provide sufficient optical power for gain saturation in the laser cavity of VCSEL 2, an erbiumdoped fibre amplifier (EDFA) was used to increase the injection power by amplifying the output power of the master laser. The wavelength conversion at $2 \mathrm{dBm}$ optical power injection was 


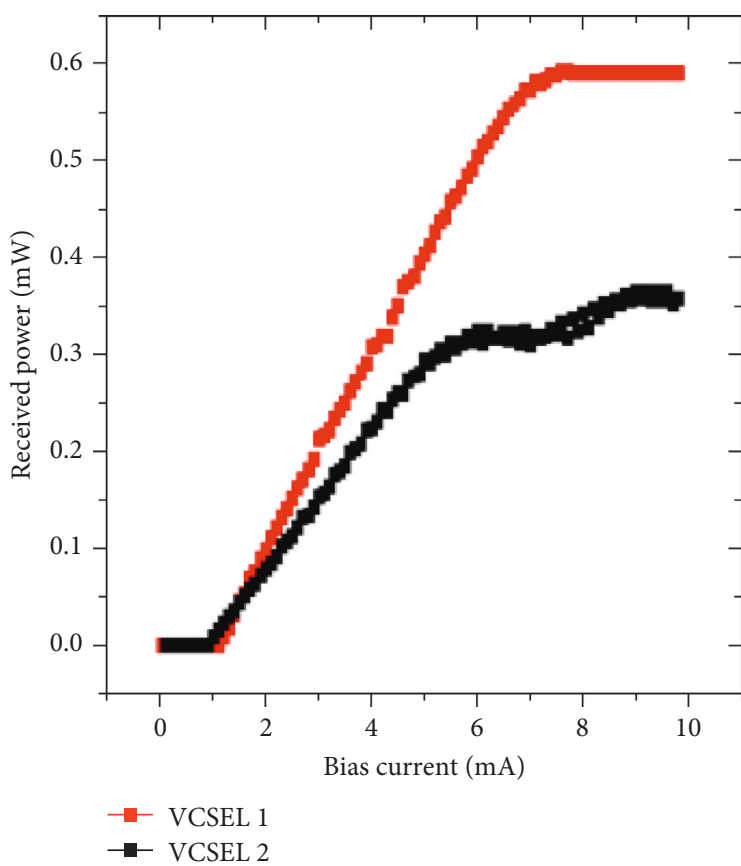

(a)

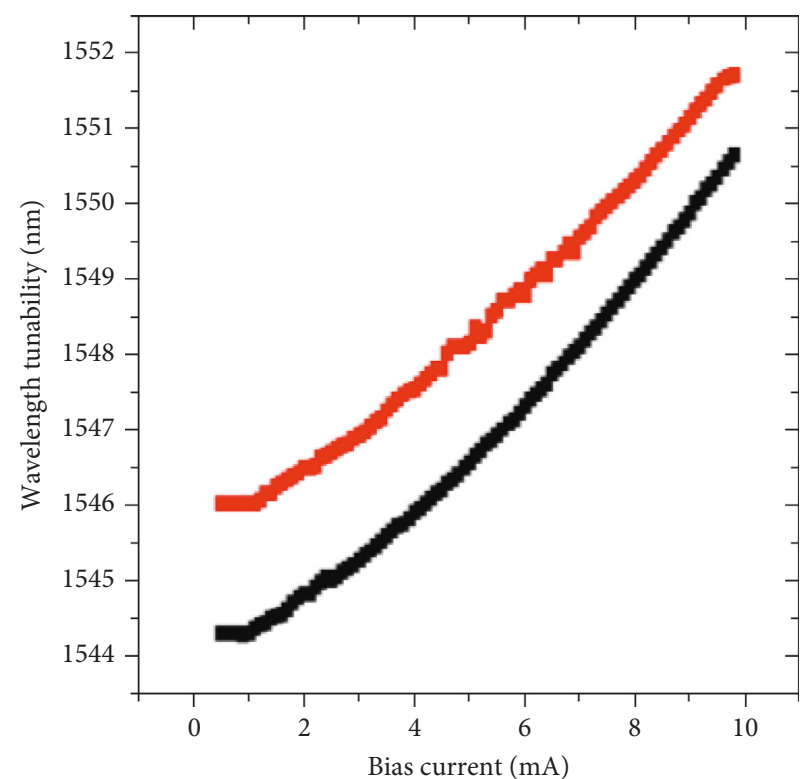

- VCSEL 1

- VCSEL 2

(b)

FIgURE 3: (a) Output power and (b) wavelength tunability of a VCSEL as a function of bias current [33].

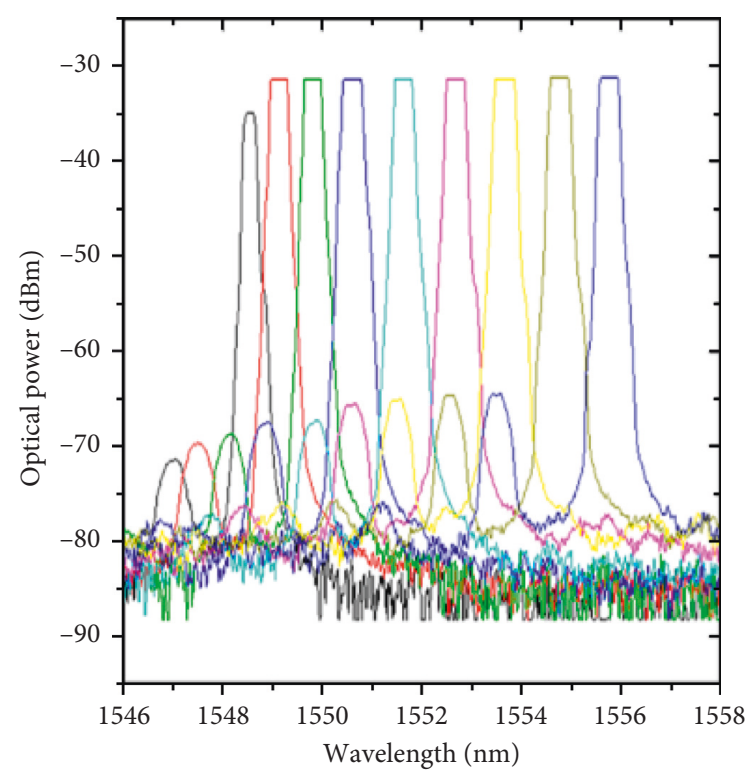

(a)

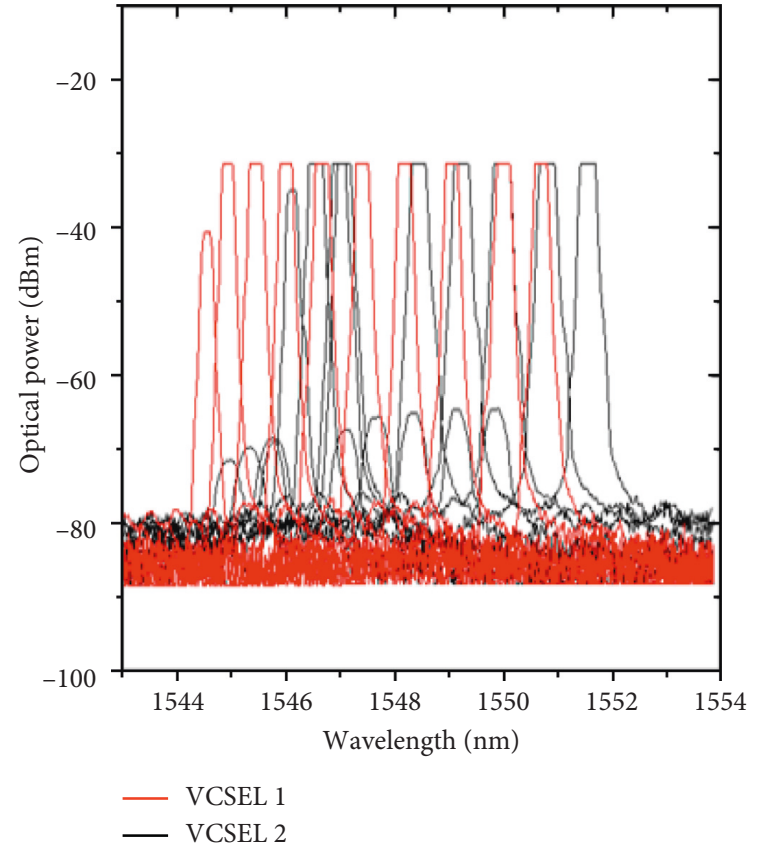

(b)

Figure 4: (a) Channel switching and (b) band overlap of VCSEL as a function of wavelength tunability [33].

considered. For accurate power monitoring, a 10/90 coupler was used, where $90 \%$ of the optical power was directly injected to the $1548.20 \mathrm{~nm}$ side mode of the slave VCSEL 2 through port 2 of the circulator and $10 \%$ was monitored via a power meter. The inverted data signal from VCSEL 2 (node 2) as a result of all-optical injection routing and wavelength assignment is emitted through port 3 of the circulator for transmission over a $25 \mathrm{~km} \mathrm{G.655} \mathrm{optical} \mathrm{fibre.} \mathrm{The} \mathrm{destination} \mathrm{(node} 3$ ) consists of a variable optical attenuator (VOA) which can be used to build a configuration representing the power loss of different fibre lightpaths similar to that of multinode networks, a positive intrinsic negative (PIN) photodiode of known error-free 


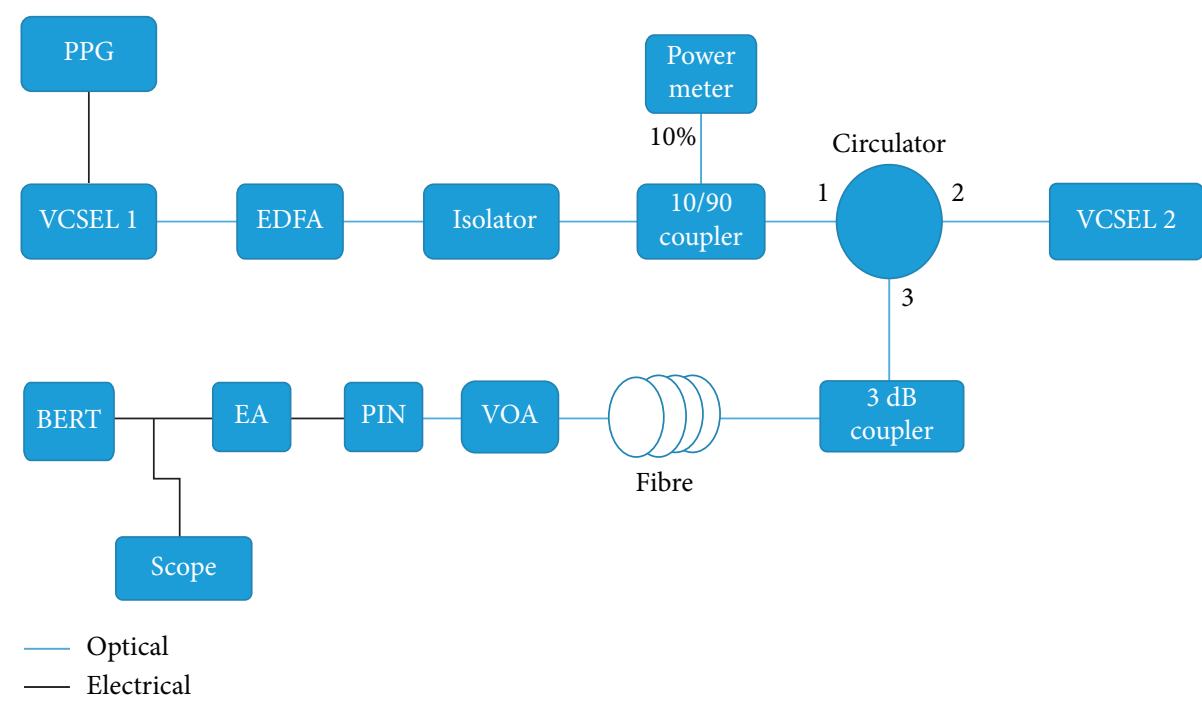

Figure 5: All optical VCSEL-to-VCSEL wavelength conversion scheme.

receiver sensitivity ( $\sim 19 \mathrm{dBm}$ at the $10^{-9}$ BER threshold), and an electrical amplifier (EA) used to amplify the signal to meet the operation requirement of the measuring and analyzing equipment. A bit error rate tester (BERT) was used to measure the extinction ratio (ER) and receiver sensitivity to analyze the quality of the data and inverted data signal at the given modulation measured as bit error rate (BER) and eye diagrams. The acceptable penalties of transmission for a given system can be quantified by analyzing the optical receiver's required power difference between the " 1 " and " 0 " levels. This is driven by the modulation which has trade-off with optical power, extinction ratio, and overshoot in eye diagrams. Overshoot is due to the change in the turn-on dynamics of a laser as the " 0 " level gets close to the threshold (bias point before lasing). For optimized bit error rate performance (good quality of service), a large difference between the power levels is required. Extinction ratio is the " 1 " and " 0 " level power ratio; when reduced, there are power penalty errors added to the system as high extinction might result in overshoot in eye diagrams. The experiment was repeated with a $25 \mathrm{~km} \mathrm{G.655} \mathrm{fibre} \mathrm{to} \mathrm{optimize} \mathrm{the} \mathrm{system} \mathrm{and}$ analyze the effects of the lightpath impairments on the system performance.

\section{Results and Discussion}

To quantify the power penalty of transmission for the system, the receiver sensitivity as a function of bias current and drive current was measured and analyzed for the master laser (VCSEL 1). This was done in a back-to-back (B2B) configuration using the VCSEL and at $25 \mathrm{~km}$ transmission over a G.655 optical fibre to analyze the effects of the lightpath impairments on the signal. A 3D surface was obtained and can be used as a domain to identify the region of optimum quality of service (receiver sensitivity) as a function of bias current of the signal carrier and the modulation conditions (drive current) of all the data to be transmitted, as shown in Figure 6.

The laser exhibits a linear optical power and bias current relationship for bias points after the threshold and before saturation; this is the safe operating regime for the VCSEL. In this regime, a finite range of possible bias and drive current combinations was used to investigate receiver sensitivity, producing a domain in the form of a 3D surface map which can be used to analyze performance of the system, as shown in Figure 6(a). For bias points in the range of 5-7 mA bias current and modulation in the range of 4-7 $\mathrm{mA}$ drive current, the best receiver sensitivity was observed. Good " 1 " and "0" level power ratios were obtained limiting VCSEL chirp and hence low penalties. The domain of the $3 \mathrm{D}$ colour receiver sensitivity surface map maintains the same shape upon $25 \mathrm{~km}$ transmission, with reduced area, as shown in Figure 6(b). At the extremities, error-free transmission is not achieved, and for a known network reach, the surface can be used to work out the penalty for the transmission; thus, the optimization technique was successfully demonstrated experimentally.

For the VCSEL, to produce an ideal signal, the drive current should be in the region just above the threshold where the VCSEL starts lasing and just below saturation of the output power (rollover). Figure 7 show the results at a fixed bias of $5 \mathrm{~mA}$, while varying the modulation drive current from $1 \mathrm{~mA}$ to $6 \mathrm{~mA}$ in $1 \mathrm{~mA}$ increments. At drive currents higher than $6 \mathrm{~mA}$, there was an error floor and a BER of $10^{-9}$ was not reached due to chirp. For the given VCSEL, the optimal modulation conditions are seen as $5 \mathrm{~mA}$ bias and $5 \mathrm{~mA}$ drive current with a receiver sensitivity of $-16.41 \mathrm{dBm}$, as shown in Figure 6. This was used to select the ideal modulation setting based on the desired quality of service (acceptable receiver sensitivity of the system) for routing and wavelength selection for error-free transmission measured at the $10^{-9} \mathrm{BER}$ threshold for data and the converted (inverted) data, as presented in Figure 7.

For VCSEL 1, data carrier had a receiver sensitivity of $-9.19 \mathrm{dBm}$ was measured for the back-to-back transmission and $-11.39 \mathrm{dBm}$ was measured for the $25 \mathrm{~km}$ transmission, resulting in a $2.2 \mathrm{~dB}$ penalty. Upon the alloptical injection onto VCSEL 2, the inverted carrier demonstrated a receiver sensitivity of -18.71 for a 


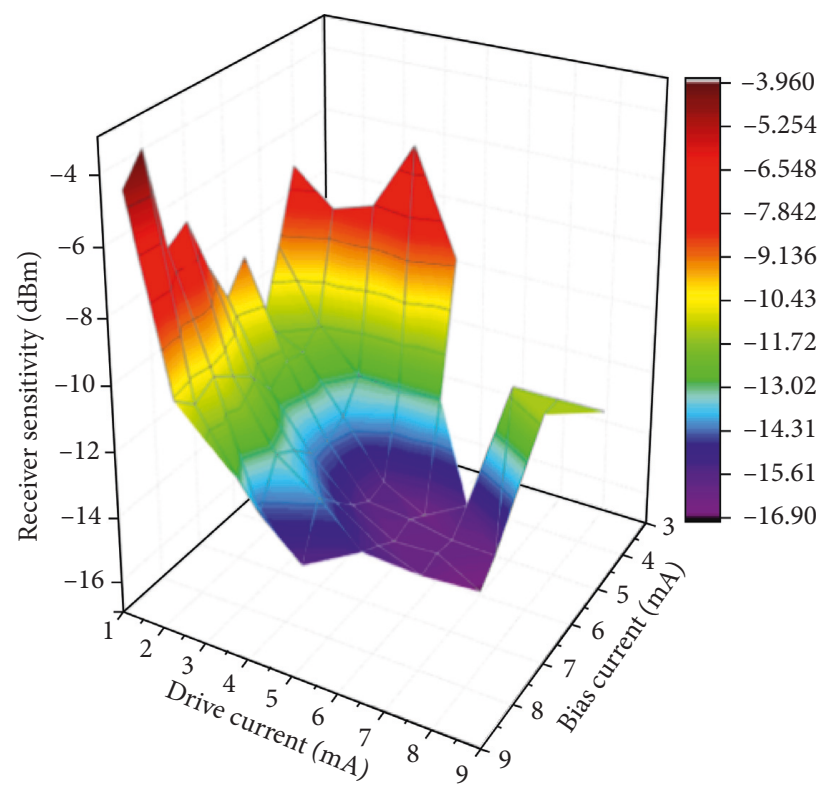

(a)

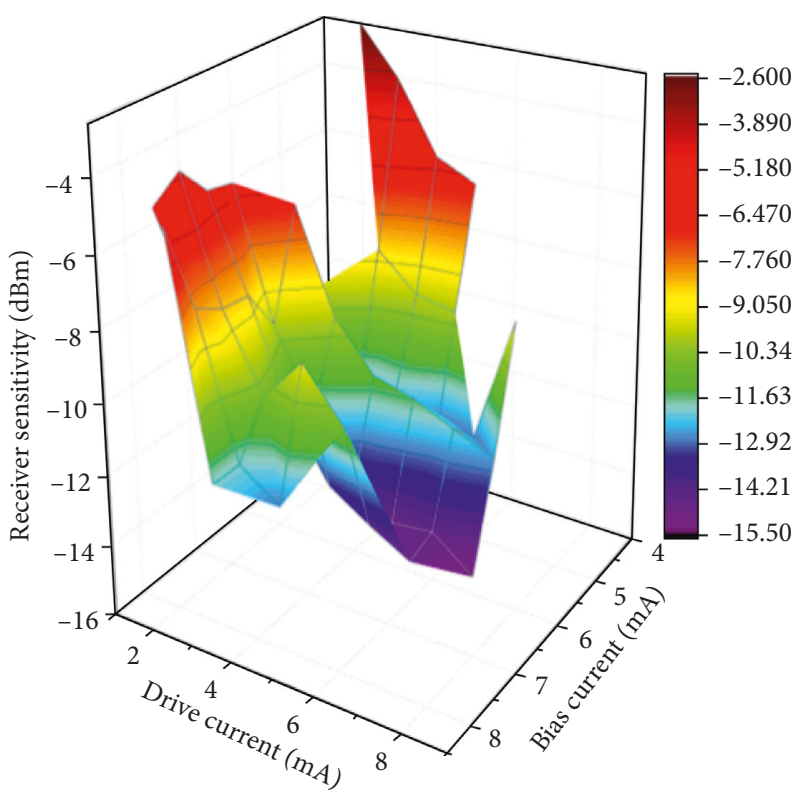

(b)

FIGURE 6: Receiver sensitivity for the VCSEL at different bias and drive currents: (a) back-to-back analysis [33] and (b) $25 \mathrm{~km}$ transmission.

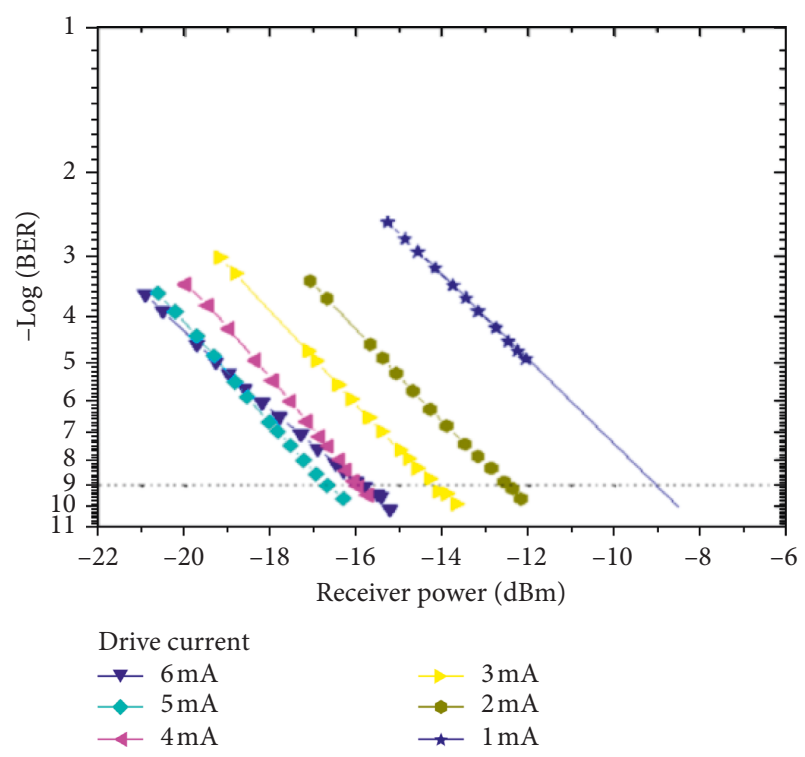

FIGURE 7: BER measurements showing receiver sensitivity at a bias of $5 \mathrm{~mA}$ and different modulation settings [33].

back-to-back transmission at the threshold and no errorfree performance for a $25 \mathrm{~km}$ inverted data transmission. For data transmission using VCSEL 1 without conversion and inversion, the eye is open, meaning the receiver can distinguish between the 0 and 1 levels and an extinction ratio of $9.136 \mathrm{~dB}$ is obtained. Upon $25 \mathrm{~km}$ transmission, the eye was distorted and the extinction ratio reduced to $8.034 \mathrm{~dB}$. For the inverted data back-to-back transmission from VCSEL 2, there is overshoot going above the 1 level with $5.012 \mathrm{~dB}$ ER obtained; this means the power levels will not be distinguishable at the receiver end, thus affecting the performance of the system. Upon $25 \mathrm{~km}$ transmission, the eye was highly distorted and closed with the extinction ratio reduced to $2.854 \mathrm{~dB}$, as shown in Figure $8(\mathrm{~b})$. Therefore, error-free transmission could not achieved regardless of the high receiver sensitivity. Hence, the extinction ratio can be used as an optimization technique; thus, it was also measured for both data back-to-back and $25 \mathrm{~km}$ transmission analyzing the eye diagrams to qualitatively examine the VCSEL performance, as shown in Figure 9.

The domain of the 3D colour extinction ratio surface map in Figure 9(a) reflects that there is a finite range of possible bias and drive currents within which the VCSEL can safely operate. Operating beyond the extreme points of the lasing threshold and the rollover point will damage the device. As expected, a high drive current results in a high extinction ratio. At low drive currents, the eye is open, meaning the receiver can distinguish between the 0 and 1 levels, although the extinction ratio is low. At high drive currents, the extinction ratio is high, but the eye is closed and there is overshoot going above the 1 level, and this means the power levels will not be distinguishable at the receiver end, thus affecting the performance of the system. At a drive current of $5 \mathrm{~mA}$, the eye is open and the extinction ratio is high, which implies that the modulation conditions are ideal for the system. The domain of the 3D colour extinction ratio surface map maintains the same shape upon $25 \mathrm{~km}$ transmission, with reduced area, as shown in Figure 9(b). With this, an effective optical performance-monitoring method for routing and wavelength assignment was experimentally demonstrated for application in flexible bandwidth multimode networks that can offer adaptive optimization of the lightpaths based on the known impairments. 


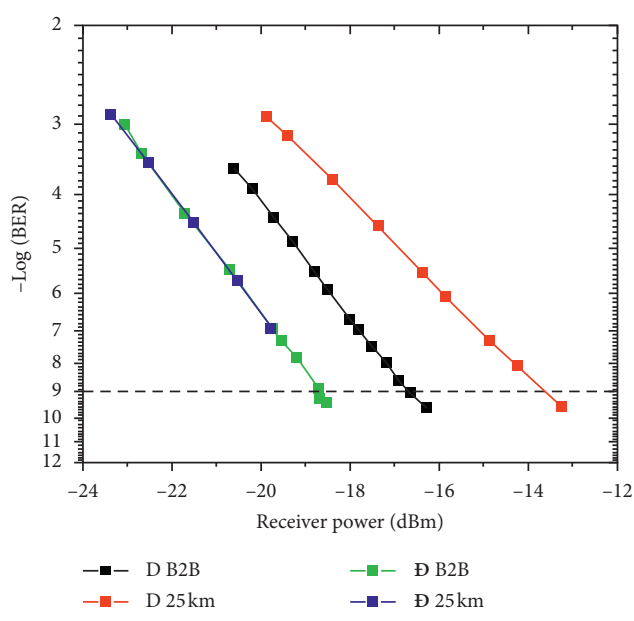

(a)
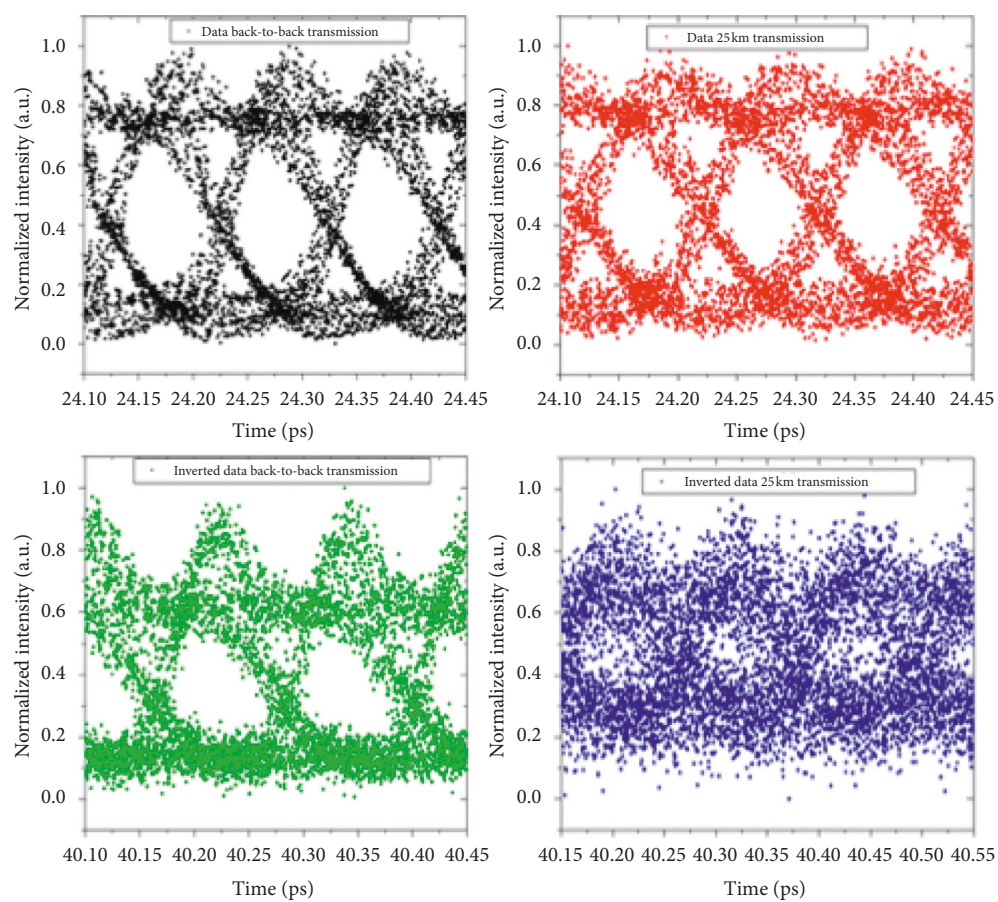

(b)

FIGURE 8: (a) BER measurements showing receiver sensitivity for data (D) and inverted data (Đ) back-to-back and $25 \mathrm{~km}$ transmission and (b) eye diagrams for each of the represented (BER) curves.

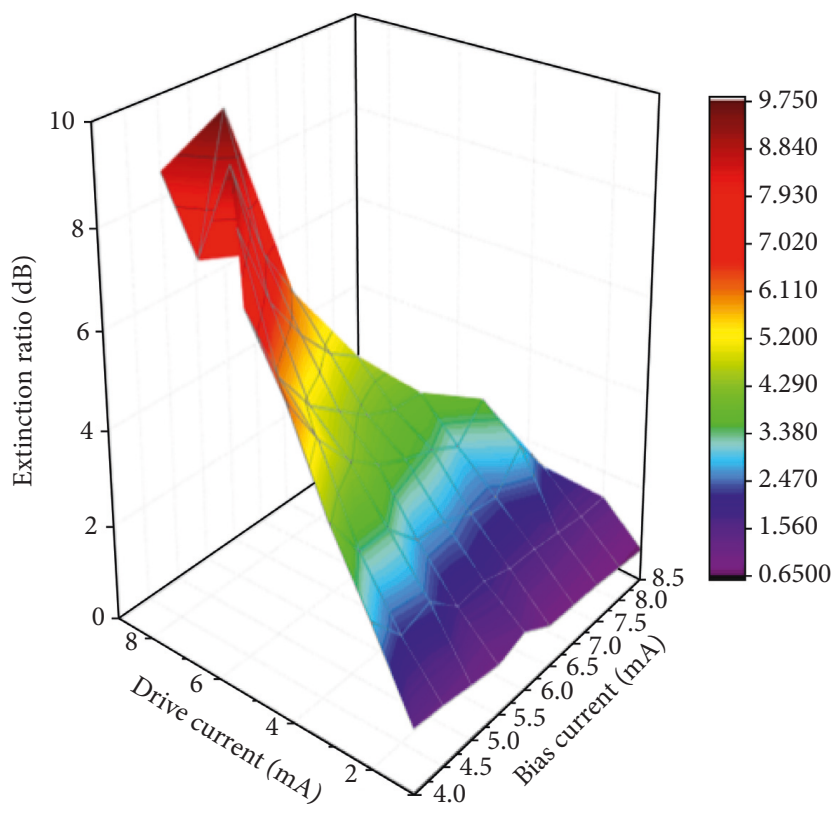

(a)

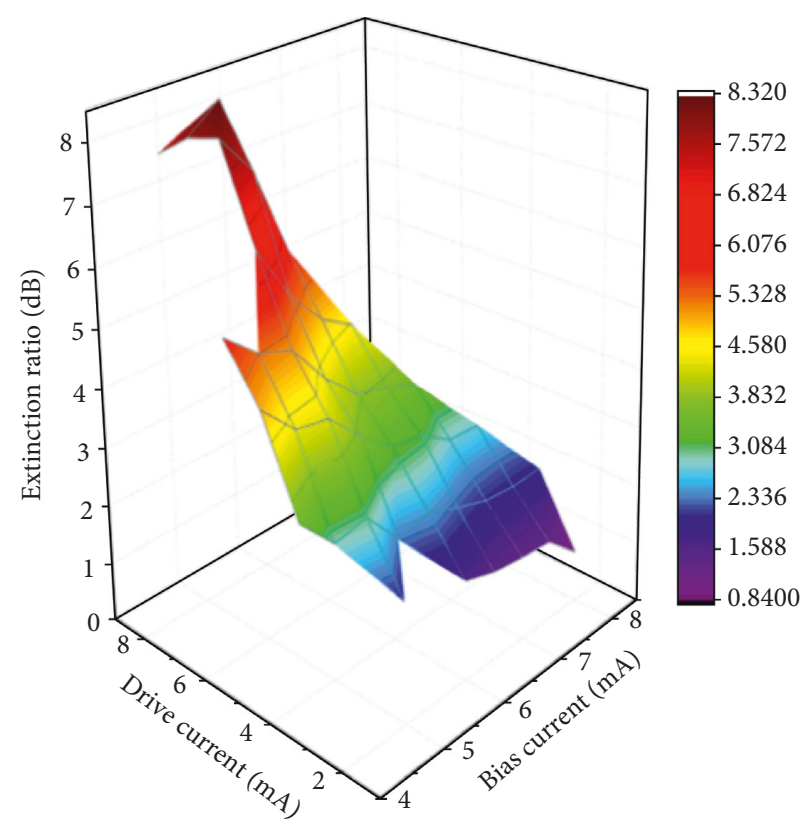

(b)

FigURE 9: Extinction ratio for the laser at different bias current points as a function of drive voltage: (a) back-to-back transmission [33] and (b) $25 \mathrm{~km}$ transmission. 


\section{Conclusion}

All-optical VCSEL-to-VCSEL injection based on the cross gain modulation has experimentally been presented for the first time to the best of our knowledge for adoption in routing and optimization of multinode flexible optical spectrum networks. A 3D domain surface map for both receiver sensitivity and extinction ratio as a function of laser bias current and modulation depth (drive current) was successfully produced and can be used for optical fibre transmission optimization. This domain can be used to find bias and drive current combinations for the VCSEL that produce reasonably high extinction ratio, high receiver sensitivity, and a wide open eye. The modulation level of a channel can be controlled to efficiently maximize the spectral bandwidth while maintaining the required quality of service as per the end user request. The optimization method was coupled with the all-optical power injection wavelength conversion through the cross gain modulation technique for efficient resource utilized and customized bandwidth demand (as per the end user request) multinode flexible spectrum networks capable of wavelength reassignment and light path rerouting with consideration of the transmission impairments. This was achieved by controlling the modulation level of a channel according to maximized spectral efficiency while maintaining the required quality of service. An optimal intensity-modulated signal was optically injected into the side mode of a VCSEL biased in the saturated output power region, subject to overshoot. Upon the resultant, wavelength conversion, error-free back-to-back and $25 \mathrm{~km}$ optical fibre transmission was successfully attained. This makes tunable lasers an essential component for the future flexible spectrum with application to wavelength switching, routing, wavelength conversion, and reconfigurable optical add-and-drop multiplexer (ROADM) for the multinode optical network; this therefore demonstrates that the performance of an all-optical fibre transport network and link management system can be optimized and used for network design, planning, and management in the centralized software-defined network controller researched for the emerging high-capacity cloud-based applications.

\section{Data Availability}

The data used to support the findings of this study are available from the corresponding author upon request.

\section{Disclosure}

This work contains an updated and developed version of the section labelled as "Proposed Spectral Defragmentation Conversion Technique" in the conference paper presentation of a manuscript in SPIE OPTO, 2019. The experimental setup has been extended for transmission over a $25 \mathrm{~km}$ G.655 fibre, and the results are analyzed in comparison to the back-to-back transmission figures presented in the manuscript. This work has been cited accordingly at DOI: $10.1117 / 12.2509981$.

\section{Conflicts of Interest}

The authors declare that they have no conflicts of interest.

\section{Acknowledgments}

The authors are grateful for research funding and support from Telkom, Dartcom, Ingoma Communication Services, CISCO, NLC, THRIP, ALC, and NRF and scholarship funding from SKA/NRF/SARAO.

\section{References}

[1] D. K. Tripathi, "Design and performance evaluations with optical comparator link," Engineering Science and Technology, an International Journal, vol. 20, no. 1, pp. 89-94, 2017.

[2] D. K. Boiyo, T. V. Chabata, E. K. R. Kipnoo, R. R. G. Gamatham, A. W. R. Leitch, and T. B. Gibbon, "Reconfigurable high-speed optical fibre networks: optical wavelength conversion and switching using VCSELs to eliminate channel collisions," Optical Fiber Technology, vol. 33, pp. 30-35, 2017.

[3] A. R. Borsali, H. A. Badaoui, M. Aichi, and W. Aichi, "Effect of channel wavelength spacing for WDM system on the quality of the transmission," International Journal of Computer Science Issues, vol. 9, no. 3, pp. 441-443, 2012.

[4] H. Zhao, Y. Zhu, F. Li, and Y. Yu, "Wavelength bistability based on optical injection in a novel tunable dual mode laser," Optics Express, vol. 24, no. 4, p. 3817, 2016.

[5] D. Amar, Performance Assessment and Modeling of Flexible Optical Networks, Optics/Photonic: Institut National des Télécommunications, Évry, France, 2016.

[6] Y. Chen and J. Mork, "Ultrahigh-frequency microwave phase shifts mediated by ultrafast dynamics in quantum-dot semiconductor optical amplifiers," IEEE Photonics Technology Letters, vol. 22, no. 12, pp. 935-937, 2010.

[7] J. Estaran, R. Rodes, T. T. Pham et al., "Quad 14 Gbps L-band VCSEL-based system for WDM migration of 4-lanes $56 \mathrm{Gbps}$ optical data links," Optics Express, vol. 20, no. 27, p. 28524, 2012.

[8] I. Tomkos, S. Azodolmolky, J. Sole-Pareta, D. Careglio, and E. Palkopoulou, "A tutorial on the flexible optical networking paradigm: state of the art, trends, and research challenges," Proceedings of the IEEE, vol. 102, no. 9, pp. 1317-1337, 2014.

[9] S. Walklin, Flexible Spectrum Networks Are Not All That Flexible, White Paper, London, UK, 2013.

[10] B. Shariati, J. M. Rivas-Moscoso, D. M. Marom et al., "Impact of spatial and spectral granularity on the performance of SDM networks based on spatial superchannel switching," Journal of Lightwave Technology, vol. 35, no. 13, pp. 2559-2568, 2017.

[11] S. Satkunarajah, K. Ratnam, and R. G. Ragel, "Efficient switch architectures for pre-configured backup protection with sharing in elastic optical networks," in Proceedings of the 7th International Conference on Information and Automation for Sustainability "Sharpening the Future with Sustainable Technology ICIAfS 2014", IEEE, Colombo, Sri Lanka, December 2014.

[12] D. J. Geisler, R. Proietti, Y. Yin et al., "The first testbed demonstration of a flexible bandwidth network with a realtime adaptive control plane," in Proceedings of the 37th European Conference and Exposition on Optical Communications, vol. 1, IEEE, Geneva, Switzerland, September 2013.

[13] P. D. Choudhury, R. S. Chauhan, P. V. Sudheer, and T. De, "Multicast routing and spectrum assignment in flexiblegrid optical networks based on light-tree sharing 
approach," in Proceedings of the-2016 15th International Conference on Information Technology (ICIT), pp. 173-179, IEEE, Bhubaneshwar, India, December 2016.

[14] D. K. Boiyo, E. K. R. Kipnoo, R. R. G. Gamatham, A. W. R. Leitch, and T. B. Gibbon, "A signal impairmentaware scheme for next-gen flexible spectrum in $10 \mathrm{~Gb} / \mathrm{s}$ VCSEL metro-access optical fibre networks," Optical Switching and Networking, vol. 25, pp. 57-62, 2017.

[15] Y. Wu, X. Xiong, Y. Zhu, J. Meng, and J. He, “All-optical wavelength conversion using optical injection induced wavelength switching in V-cavity laser," in PIERS Proceedings, pp. 915-919, Gangzhou, China, August 2014.

[16] S. L. Jansen, H. Chayet, E. Granot et al., "Wavelength conversion of a $40 \mathrm{gb} / \mathrm{s}$ NRZ signal across the entire C-band by an asymmetric Sagnac loop," IEEE Photonics Technology Letters, vol. 17, no. 10, pp. 2137-2139, 2005.

[17] S. Mazzucato, K. Schires, A. Hurtado, M. J. Adams, I. D. Henning, and N. Balkan, "Conversion for VCSEL-toVCSEL inversion inversion and logic," IEEE Photonics Journal, vol. 4, no. 3, pp. 817-824, 2012.

[18] D. Cotter, "Optical communication systems," Optica Acta: International Journal of Optics, vol. 31, no. 8, pp. 847-848, 1984.

[19] L. A. Coldren, G. A. Fish, Y. Akulova, J. S. Barton, L. Johansson, and C. W. Coldren, "Tunable semiconductor lasers: a tutorial," Journal of Lightwave Technology, vol. 22, no. 1, pp. 193-202, 2004.

[20] Application Note AN-2135, Application Note-Modulating VCSELs, Finisar, Sunnyvale, CA, USA, 2015.

[21] NMMU Broadband Centre of Excellence, Confidential Cisco, Site Requirements Specification (SRS) for SKA-NMMU Broadband Centre of Excellence Research Network DWDM Lab Version 3, Cisco Systems Advanced Services, Johannesburg, South Africa, 2017.

[22] F. Zhang and R. Casellas, Requirements for GMPLS Control of Flexible Grids, Internet Engineering Task Force, Fremont, CA, USA, 2012.

[23] L. Velasco, M. Klinkowski, M. Ruiz, and J. Comellas, "Modeling the routing and spectrum allocation problem for flexgrid optical networks," Photonic Network Communications, vol. 24, no. 3, pp. 177-186, 2012.

[24] G. Bruno, N. Sambo, P. Iovanna, P. Castoldi, G. Bottari, and F. Cugini, "Lightpath provisioning in wavelength switched optical networks with flexible grid," in Proceedings of the 2011 37th European Conference and Exposition on Optical Communications, IEEE, Geneva, Switzerland, September 2013.

[25] OFS Marketing Communication, TrueWave ${ }^{\circledR}$ RS Optical Fiber, OFS, Chicago, IL, USA, 2018.

[26] OFS Marketing Communications and A Furukawa Company, "TrueWave ${ }^{\circledR}$ LA low water peak optical fiber," in Optimized for $10 \mathrm{G}$ Networks in Metro, Regional, and Long-Haul Applications, OFS, Chicago, IL, USA, 2017.

[27] International Telecommunication Union (ITU-T), G.973.2 (04/2011), Multichannel DWDM Applications with Single Channel Optical Interfaces for Repeaterless Optical Fibre Submarine Cable Systems, International Telecommunication Union, Geneva, Switzerland, 2011.

[28] F. Cugini, G. Meloni, F. Paolucci et al., "Demonstration of flexible optical network based on path computation element," Journal of Lightwave Technology, vol. 30, no. 5, pp. 727-733, 2012.

[29] A. Farrel, D. King, Y. Li, and F. Zhang, Generalized Labels for the Flexi-Grid in Lambda Switch Capable (LSC) Label
Switching Routers, RFC 7699, Internet Engineering Task Force, Fremont, CA, USA, 2015.

[30] C. R. Doerr, S. Chandrasekar, L. L. Buhl et al., "Repeaterless transmission with negative penalty over $285 \mathrm{~km}$ at $10 \mathrm{~Gb} / \mathrm{s}$ using a chirp managed laser," IEEE Photonics Technology Letters, vol. 17, no. 11, pp. 2454-2456, 2005.

[31] T. Ohtsuki, T. Yatsu, and M. Matsuura, "Regenerative wavelength conversion of PAM-4 signals using XGM with blue-shift filtering in a QD-SOA," in Proceedings of the 2017 Conference on Lasers and Electro-Optics Pacific Rim (CLEO$P R)$, pp. 1-3, IEEE, Singapore, August 2017.

[32] I. Z. Mazlan, M. H. A. Wahid, A. F. Hassan, M. A. M. Azidin, and N. F. M. Isa, "Cross gain modulation (XGM) based on wavelength conversion using semiconductor optical amplifier and filter," International Journal of Microwave and Optical Technology, vol. 9, no. 1, pp. 115-118, 2014.

[33] P. P. Dlamini, G. M. Isoe, D. K. Boiyo, A. W. R. Leitch, and T. B. Gibbon, "Cascaded VCSEL-to-VCSEL all-optical injection for spectral defragmentation and switching using optical transmitter cross gain modulation," in Proceedings of the Metro and Data Center Optical Networks and Short-Reach Links II, vol. 10946, San Francisco, CA, USA, Febraury 2019.

[34] D. K. Boiyo, G. M. Isoe, R. R. G. Gamatham, A. W. R. Leitch, and T. B. Gibbon, "A $1550 \mathrm{~nm}$ all-optical VCSEL-to-VCSEL wavelength conversion of a $8.5 \mathrm{~Gb} / \mathrm{s}$ data signal and transmission over a $24.7 \mathrm{~km}$ fibre," Proceedings of SPIE-The International Society for Optical Engineering, vol. 10036, 2017. 

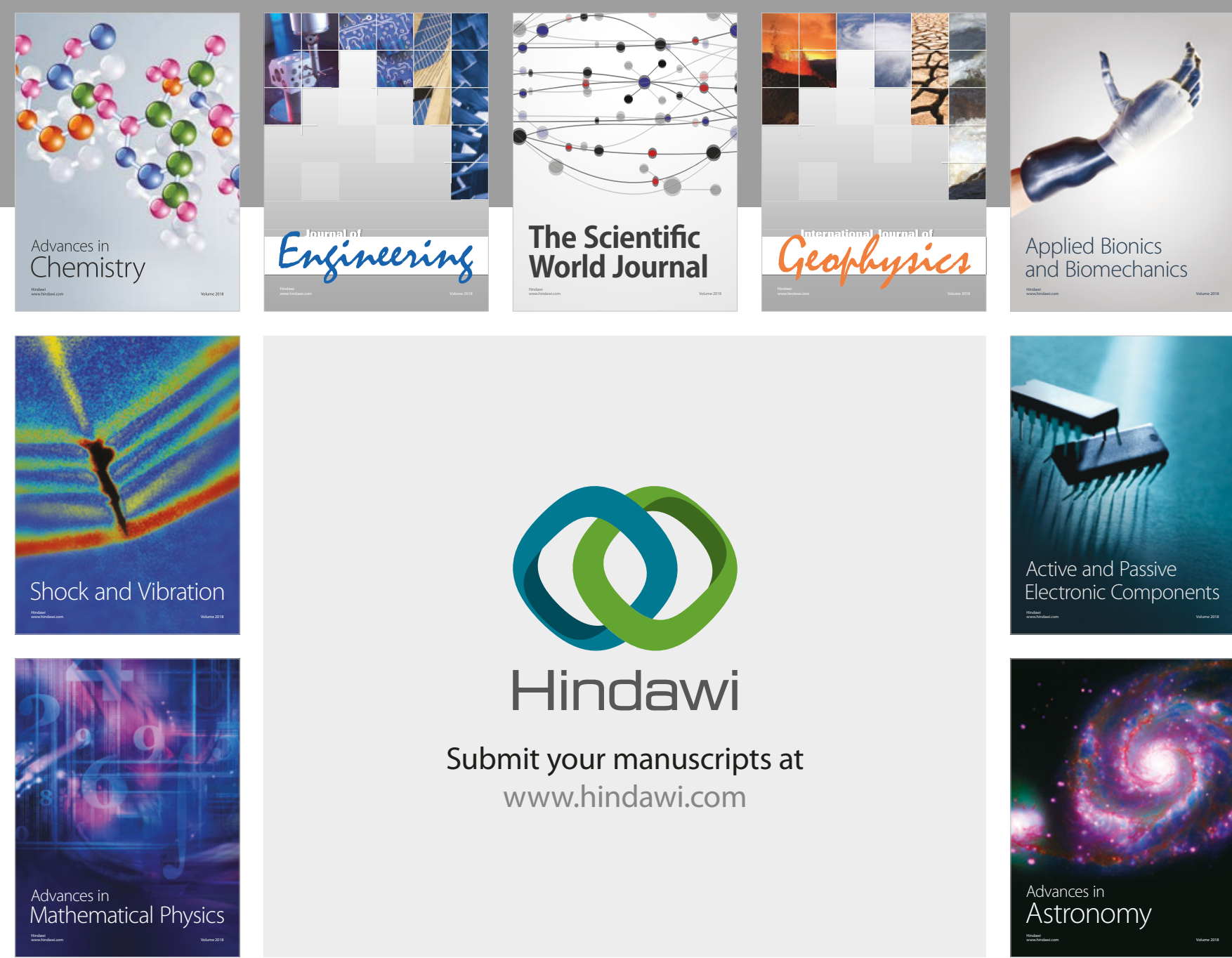

Submit your manuscripts at

www.hindawi.com

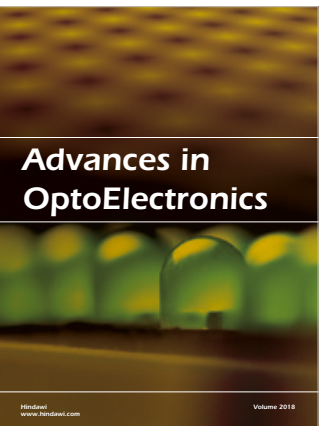

\section{Rotcting Machinery}
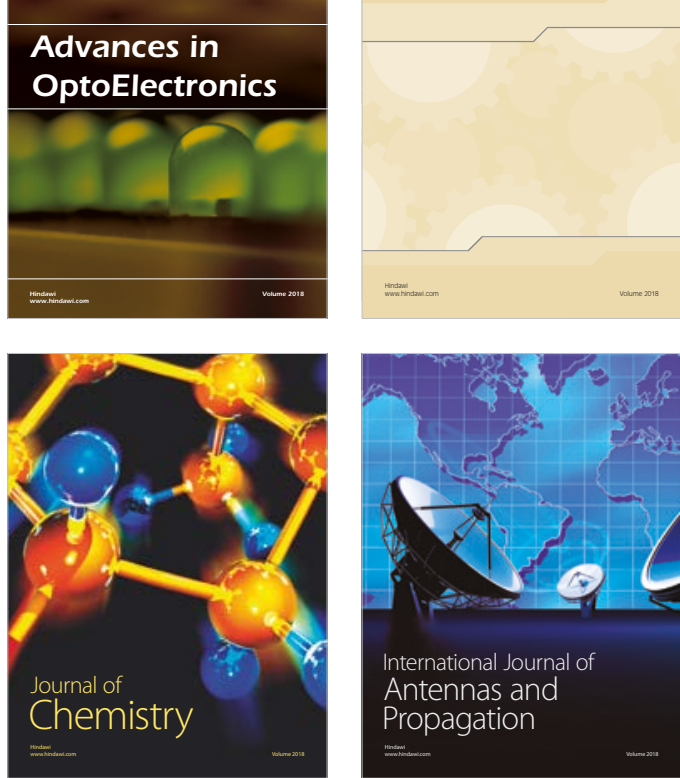

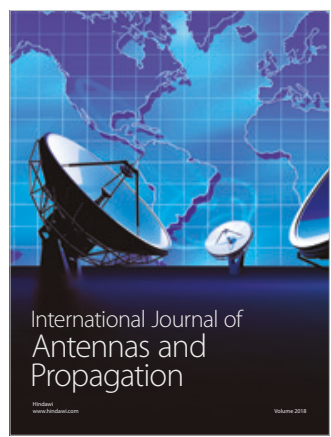

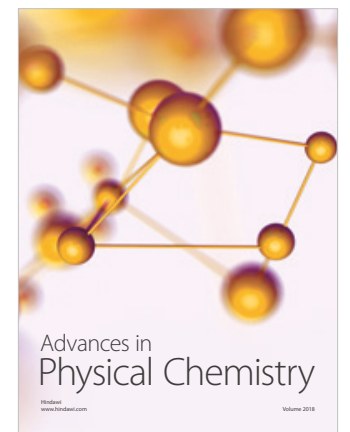

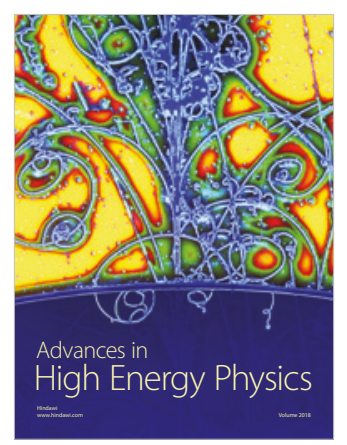

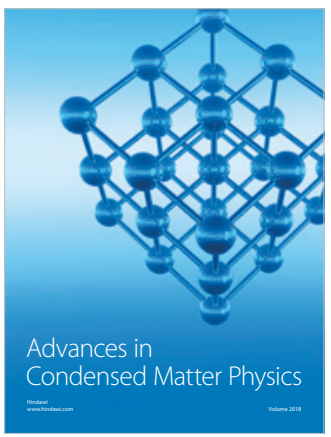

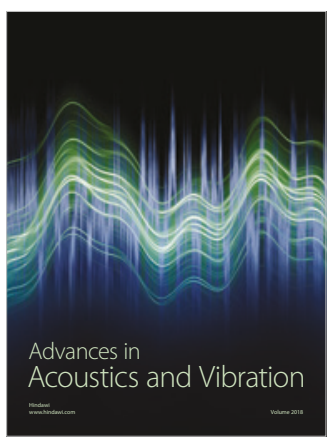

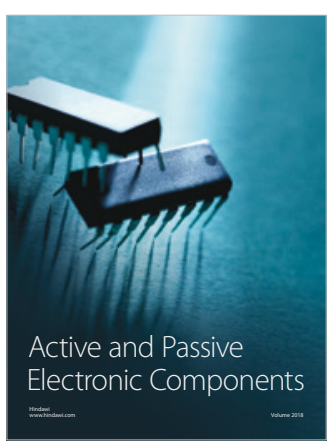
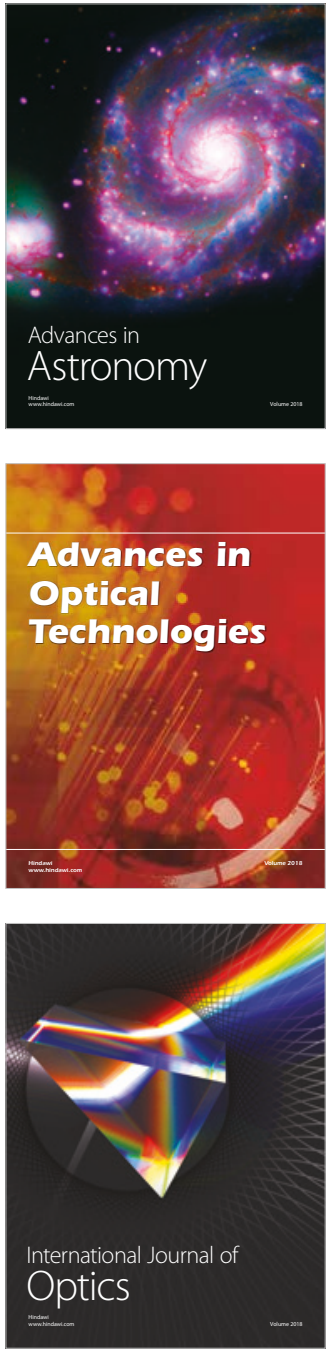